\title{
Direct time-domain determination of electron-phonon coupling strengths in chromium
}

\author{
J. Wingert $\odot,{ }^{1, *}$ A. Singer, ${ }^{1, \dagger}$ S. K. K. Patel, ${ }^{1,2}$ R. Kukreja, ${ }^{1,2,3}$ Matthieu J. Verstraete, ${ }^{4}$ Aldo H. Romero, ${ }^{5}$ V. Uhlîr $\odot,{ }^{2,6}$ \\ S. Festersen, ${ }^{7}$ D. Zhu, ${ }^{8}$ J. M. Glownia ${ }^{8}$ H. T. Lemke, ${ }^{8,9}$ S. Nelson, ${ }^{8}$ M. Kozina, ${ }^{8}$ K. Rossnagel $\odot,{ }^{7,10,11}$ B. M. Murphy ${ }^{\circledR},{ }^{7,10}$ \\ O. M. Magnussen, ${ }^{7,10}$ E. E. Fullerton, ${ }^{2}$ and O. G. Shpyrko ${ }^{1}$ \\ ${ }^{1}$ Department of Physics, University of California, San Diego, La Jolla, California 92093, USA \\ ${ }^{2}$ Center for Memory and Recording Research, University of California, San Diego, La Jolla, California 92093, USA \\ ${ }^{3}$ Department of Materials Science and Engineering, University of California, Davis, 1 Shields Avenue, Davis, California 95618, USA \\ ${ }^{4}$ nanomat/Q-MAT/CESAM, Université de Liège, and European Theoretical Spectroscopy Facility, allée du 6 août, 19, B-4000 Liège, Belgium \\ ${ }^{5}$ Department of Physics and Astronomy, West Virginia University, Morgantown, West Virginia 26505-6315, USA \\ ${ }^{6}$ CEITEC BUT, Brno University of Technology, Purkyňova 123, CZ-612 00 Brno, Czech Republic \\ ${ }^{7}$ Institute for Experimental and Applied Physics, Kiel University, D-24098 Kiel, Germany \\ ${ }^{8}$ LCLS, SLAC National Accelerator Laboratory, Menlo Park, California 94025, USA \\ ${ }^{9}$ Paul Scherrer Institut, Forschungsstrasse 111, CH-5232 Villigen, Switzerland \\ ${ }^{10}$ Ruprecht Haensel Laboratory, Kiel University, D-24098 Kiel, Germany \\ ${ }^{11} 12$ Deutsches Elektronen-Synchrotron DESY, D-22607 Hamburg, Germany
}

(Received 12 October 2019; revised 18 May 2020; accepted 11 June 2020; published 2 July 2020)

\begin{abstract}
We report the results of an ultrafast, direct structural measurement of optically pumped phonons in a $\mathrm{Cr}$ thin film using ultrashort $\mathrm{x}$-ray pulses from a free-electron laser. In addition to measuring and confirming the known long-wavelength dispersion relation of $\mathrm{Cr}$ along a particular acoustic branch, we are able to determine the relative phase of the phonons as they are generated. The Cr sample exhibits two generation mechanisms for the phonons: the releasing of a preexisting charge density wave at higher frequencies, and the creation of an acoustic strain pulse via laser heating that dominates at lower frequencies. For the latter mechanism, we are able to measure the frequency dependence of the time required to generate the phonons. To explain the observed magnitude and slope of the delays, we perform first-principles simulations in the framework of density functional perturbation theory and $a b$ initio molecular dynamics to fit anharmonic phonon models. These results show that the wave-vector dependence of the electron-phonon coupling is the driving mechanism behind the delay times: Phase-space limitation leads to higher times near the zone center. The absolute magnitudes of the delay times measured are found to be much shorter than the equilibrium electron-phonon coupling times we compute, indicating that the coupling strength is greatly enhanced when the electronic system is out of equilibrium with the lattice, as has been seen in bismuth and other systems.
\end{abstract}

DOI: 10.1103/PhysRevB.102.041101

Understanding the interactions between electrons and phonons in condensed matter systems is of both fundamental and practical importance. Until quite recently, the primary methods for studying phonons in solids have been inelastic scattering techniques. For several decades, inelastic neutron scattering (INS) has been used extensively to characterize dispersion relations in a wide range of materials, including chromium [1,2]. More recently, frequency-domain studies using inelastic x-ray scattering (IXS) have also driven advances in our understanding of phonons in condensed matter systems [3]. Ultrafast electron diffraction has been employed to measure direct structural responses to optical excitation, but aside from one very recent work [4], this has been limited to studying the time evolution of the central Bragg peak [5-7]. The direct time-domain measurement of struc-

\footnotetext{
*jcwingert@physics.ucsd.edu

${ }^{\dagger}$ Present address: Department of Materials Science and Engineering, Cornell University, Ithaca, NY 14853.
}

tural changes in solids using $\mathrm{x}$ rays on ultrafast $(<1 \mathrm{ps})$ timescales has only become possible in the last few years. Although x-ray pump-probe techniques have been used for some time at synchrotron sources [8], it is the extremely short pulses ( $<50 \mathrm{fs}$ ) generated by free-electron lasers (FELs) that have pushed these techniques into the ultrafast regime, and allowed for the direct detection of picosecond lattice oscillations [9-16]. It has been possible for some time to generate optical laser pulses of similarly short duration. For this reason, ultrafast pump-probe experiments using a laser pump and a laser probe were possible long before $\mathrm{x}$ rays could be used as a probe to study the same timescales. It was these all-optical pump-probe studies that allowed for the determination of electron-phonon relaxation times in various materials, including $\mathrm{Cr}$ [17]. However, these studies typically measure ultrafast changes in reflectivity, and are thus only sensitive to the rate of electron cooling. Measuring acoustic pulse propagation via reflectivity is also possible, but this approach effectively integrates the phonon response across all frequencies [18]. Now, with the ultrashort $\mathrm{x}$-ray pulses 
produced by FELs, it is possible to directly measure while the electronic system cools, and access the generation rate of phonons for a variety of wave vectors and frequencies. Here, we induce phonons in a $\mathrm{Cr}$ thin film with ultrafast optical excitation, and determine the phonon amplitudes and phases in momentum space using x-ray diffraction. We are able to measure the phase of the phonons by capturing the $\mathrm{x}$-ray interference between the Laue fringes and the phonon scattering. This measurement of phonon phases is differentboth conventional and more recent methods for measuring phonons (INS, IXS, ultrafast optical pump probe, ultrafast electron diffraction, and even x-ray diffraction) either have no access to the phase of the phonon, or have not measured it across a range of frequencies as we do here.

When an optical pulse is incident on a metal, it is primarily absorbed by the conduction electrons that lie within the optical penetration depth (about $20 \mathrm{~nm}$ at a wavelength of $800 \mathrm{~nm}$ in $\mathrm{Cr}$ ). In this experiment, the laser pulse is incident on the sample at an angle of roughly $30^{\circ}$, so that the penetration depth normal to the film surface is reduced to about $10 \mathrm{~nm}$. The hot electrons then propagate further into the metal (while energy and momentum exchange within the electron system takes place as well). The depth to which these hot electrons can propagate before cooling is determined by the strength of electron-phonon coupling in the particular material, and is about $15 \mathrm{~nm}$ in $\mathrm{Cr}$ [17]. Once the energy is transferred to the lattice, it creates a displacement field and associated strain profile over the same length scale. If this distance is relatively short (as it is in $\mathrm{Cr}$ ), the central frequency of the resulting acoustic pulse will be relatively high. In bulk $\mathrm{Cr}$, which demonstrates strong electron-phonon coupling, this is about $50 \mathrm{GHz}$ [17], whereafter the amplitudes of the oscillations decay monotonically. In a film such as the one studied here, of thickness similar to the optical penetration depth, the shape of the acoustic pulse may be somewhat altered. Here, our goal is not to attempt to recreate the precise spatiotemporal shape of the acoustic pulse we generate, but rather to characterize the generation of the individual phonons it comprises. It should be noted that there are other mechanisms by which optical light can drive acoustic oscillations in solids, such as displacive excitation of coherent phonons (DECP) [16,19-22], but as shown below, our data clearly indicate that acoustic strain pulse formation is the primary phonon generation mechanism at work in our experiment, as expected in metals [23].

In materials that exhibit charge density wave (CDW) order, such as our Cr film, there is another mechanism by which acoustic phonons can be created. The band structure of $\mathrm{Cr}$ results in spin density wave electronic ordering of the free electrons, with a period that is incommensurate with the lattice [24]. This results in a CDW whereby the equilibrium positions of the atoms are sinusoidally modulated. This is essentially a frozen phonon. When an optical pulse with sufficient energy is absorbed by the electrons, the electronic order is destroyed, and there is an abrupt shift in the potential energy environment of the atoms. This thaws the frozen phonon and allows it to propagate $[13,25]$. Unlike the optical heating mechanism that drives phonons in all metals, there is effectively no delay for this process - the phonon begins to ring immediately after the electronic temperature rises. We show that, rather than complicating our analysis, this "CDW mechanism" of phonon generation provides a reference to which we can compare those generated via the "thermal mechanism." In comparison to our previous study of the same sample at cryogenic temperatures [13], the CDW observed here at room temperature has a lower spatial frequency and reduced amplitude, which are shifted by an amount consistent with previous studies [24]. We want to stress that previous works by Singer $e t$ al. studied this same Cr sample [13,25,26], focusing exclusively on the CDW, and for that reason we do not include a more in-depth discussion of this mechanism here.

A DC-sputtered, 28-nm $\mathrm{Cr}$ thin film, grown on a $\mathrm{MgO}$ substrate and annealed at $500^{\circ} \mathrm{C}$ was used for this study. The crystal orientation of the film and $\mathrm{MgO}$ substrate are both [001], as determined by x-ray diffraction. The diffraction pattern around the central (002) Bragg peak was recorded at the Advanced Photon Source (APS), using a point detector [25]. The results of this measurement are shown in Fig. 1 in black. Due to the highly uniform thickness of the film, well-resolved Laue fringes with strong intensity are clearly visible out to high order.

Using the XPP station at the Linac Coherent Light Source (LCLS) [11], the diffraction from this sample was measured with a two-dimensional (2D) detector, the Cornell-SLAC hybrid Pixel Array Detector (CSPAD). Throughout the experiment, the detector and incident beam were held fixed, at an x-ray scattering angle of $2 \theta=60^{\circ}$. By rotating the sample slightly, we were able to record images corresponding to different cuts through the fringes along the Bragg rod. Three such images are also shown in Fig. 1 alongside the synchrotron data. Due to mosaic spread in the sample, several Laue fringes are visible simultaneously at a given sample angle [13]. All measurements were carried out at room temperature. It should be emphasized that the high intensity of the Laue fringes out to large $Q$ values is what allows us to measure a strong signal in regions of momentum space that would typically exhibit only weak diffuse scattering.

For the pump-probe measurements, the sample was optically pumped with an $800-\mathrm{nm}, 40$-fs duration laser pulse. The fluence delivered to the sample was about $5 \mathrm{~mJ} / \mathrm{cm}^{2}$, as estimated from the shift in the central Bragg peak (this fluence was consistent with waveplate calibration scans from a $\mathrm{MgO}$ reference sample for the angle we chose). Diffraction patterns were then collected by probing the sample with a 15 -fs $\mathrm{x}$-ray pulse $(\lambda=0.14 \mathrm{~nm})$ at delay times ranging from -2 to $10 \mathrm{ps}$. This was done at several sample angles, so that, on the "low$Q$ " side relative to the Bragg peak, data were collected out to the tenth-order Laue fringe. The laser pump and x-ray probe had beam diameters of 0.5 and $0.2 \mathrm{~mm}$, respectively. Conveniently, although the relative brightness of each fringe changes dramatically when the sample is rotated, the fringe positions on the detector move by less than a pixel. This allows us to combine multiple images directly, without making geometric corrections. After being summed, the combined images are then integrated vertically (with respect to the images in Fig. 1). This is done for the images collected at all possible delay times, yielding intensity values as a function of $Q$ and time. In Fig. 2(a), a combined image of all measurements made on the low- $Q$ side of the Bragg peak is shown. The intensities have been normalized by the $t<0$ values to make the oscillations visible for all fringes. 

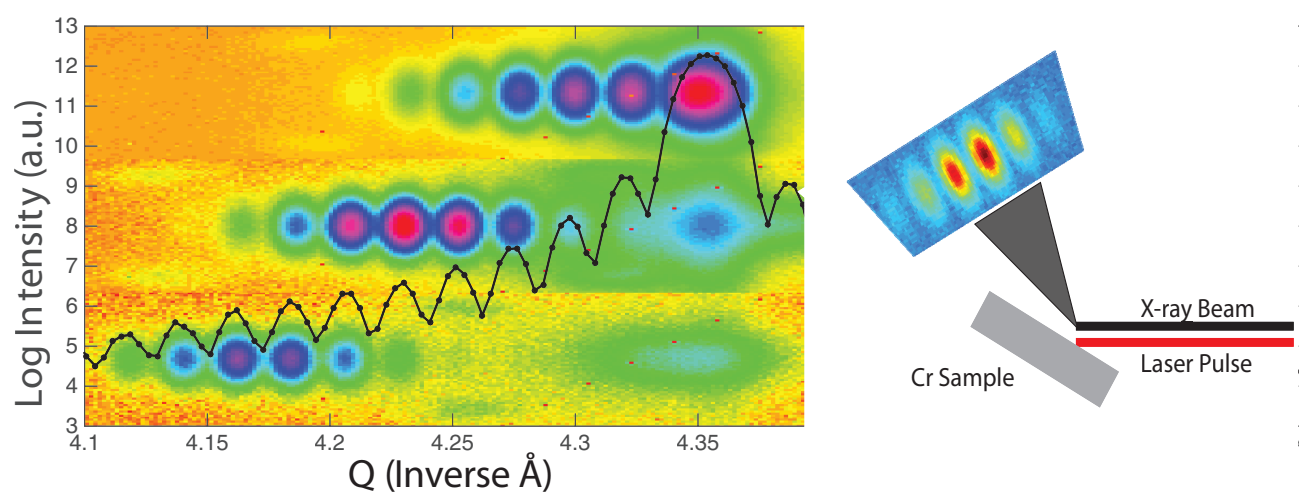

FIG. 1. The axes and black data points are from data taken at APS. The central peak is the [002] Bragg peak for Cr, and the Laue fringes around it have a periodicity corresponding to the $28-\mathrm{nm}$ film thickness. Shown behind this are three separate area detector images taken at LCLS, corresponding to different cuts through these fringes on the Bragg rod, achieved by rocking the sample. On the right, a simple schematic of the LCLS experimental setup is shown.

To extract a one-dimensional intensity signal versus time for the individual fringes, a range of pixel values along $Q$ were identified that clearly do not overlap with neighboring fringes, yet still fully contain the fringe as it shifts in $Q$ slightly during the measurement (due to a slow oscillation of the central Bragg peak). These regions are then integrated over this small range of $Q$ values, which results in a one-dimensional vector of intensities as a function of time delay. These resulting time series are shown in Fig. 2(b). After the signals are obtained for each fringe, we take their Fourier transforms in time. We then fit Gaussians to the main peaks identified in the amplitudes of the transforms. The means and standard deviations obtained from each of these fits serve as our frequency data points and error bars, respectively. These are plotted versus the $Q$ value associated with each fringe (in units relative to $Q_{\mathrm{Bragg}}$ ) in Fig. 3. A linear regression is performed to determine the

(a)

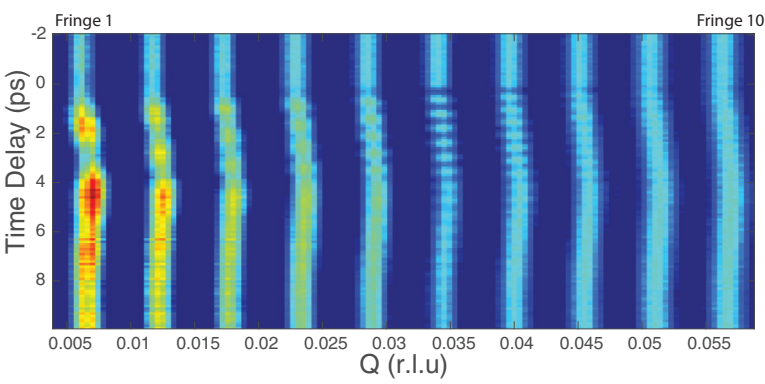

(b)

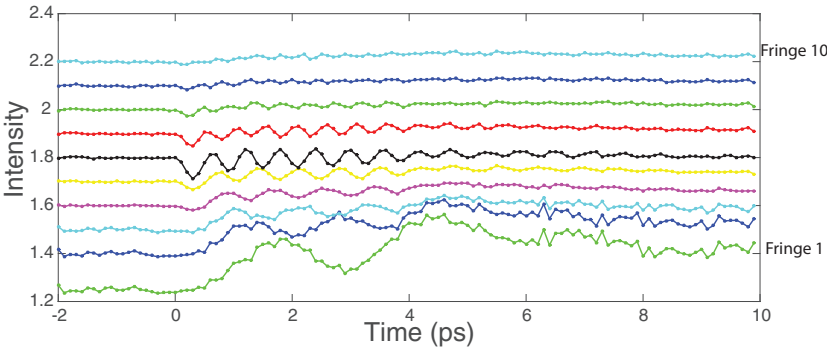

FIG. 2. (a) Slices through the Bragg rod for the low- $Q$ fringes combined in one image, integrated vertically with respect to Fig. 1, normalized by the $t<0$ values, and plotted vs time (Bragg peak not shown). (b) The extracted 1D signals for the fringes shown in (a). slope of the best fit line for these points. As we are in the long-wavelength limit, where phonons propagate at a velocity $v=\omega / K$ [27], the slope of this line should give the speed of sound, for longitudinal waves in the [001] direction. This is found to be $7.45 \mathrm{~nm} / \mathrm{ps}$ in our sample, which is in good agreement with data from inelastic neutron scattering [1]. The amplitudes of the Fourier transforms are also plotted in Fig. 3 (heights of Gaussians fit to the Fourier transforms of the time signals). The enhanced oscillation amplitude of the signal for fringe 6 and its neighbors is consistent with the existence of a residual charge density wave with a periodicity centered near fringe 6 , which persists above the Néel temperature for our thin film $[13,24]$. The amplitudes can be fit well with a Gaussian (as used to model the Laue fringe envelope from surface roughness in Ref. [26]) summed with a Lorentzian for the CDW (a Gaussian CDW peak gives a slightly lower quality fit). An important feature of the data is the reduced amplitude of the oscillations that occurs after about 4 ps (see Fig. 2). This corresponds to the time it takes sound to

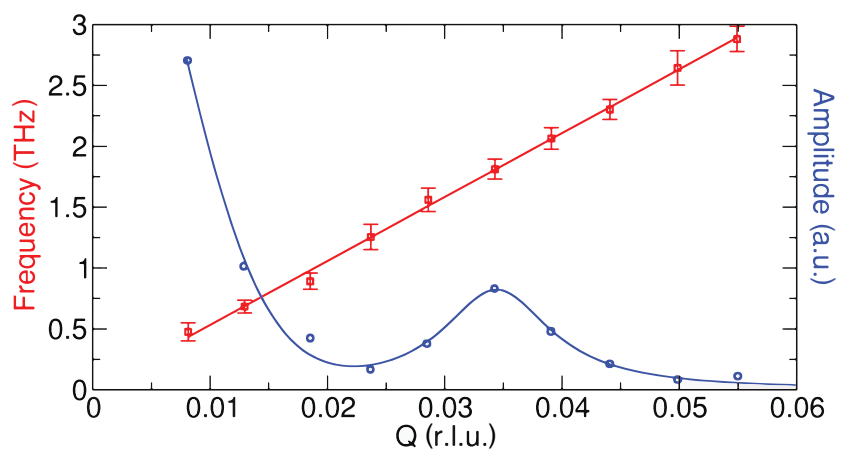

FIG. 3. The dispersion curve points for all measured fringes on the low- $Q$ side are shown, along with their amplitudes. The red line shown is a best fit through all the frequency data points, whose slope gives the longitudinal speed of sound in the $\mathrm{Cr}$ film normal to the surface, which is found to be $7.45 \mathrm{~nm} / \mathrm{ps}$ for our sample. The enhancement of fringes 5,6 , and 7 is attributed to residual CDW ordering that persists above the Néel temperature. The amplitude fit (blue line) is described in the text. 
propagate across the film at the velocity we observed, and confirms that we indeed generate an acoustic pulse at the free interface which then travels across the film, before being reflected by the $\mathrm{MgO}$ substrate.

As we directly measure the phonon oscillations in the time domain, we are sensitive to their phase. In order to determine the relative phase for each phonon frequency measured, we calculate the locations of the first two maxima of the oscillations in time, using a smoothing filter. We then divide these times by the period of the signal, which is determined from the Fourier analysis previously discussed, and subtract off an appropriate integer multiple of $2 \pi$. As can be seen directly from the raw data in Fig. 2(b), the first three fringes have a qualitatively different phase relation than the other fringes. Moreover, one would expect the amplitudes of the signals to decrease monotonically in $Q$, as occurs in bulk $\mathrm{Cr}$ after about $50 \mathrm{GHz}$, a far lower frequency than any measured here. Instead, the amplitudes begin to rise again after the fourth fringe. We attribute these deviations from expected bulk behavior to the presence of two distinct phonon generation mechanisms. For the higher-order fringes, the response is driven by the presence of a CDW, which begins to ring immediately after the electrons are heated. For the lowest-order fringes, the generation mechanism is the typical heating/strain pulse mechanism. These phonons are only generated on the timescale of the hot-electron-phonon relaxation time. To obtain a simple measure of the delay times for phonon generation, we note that the first three fringes appear to have a cosine phase, and the higher-order fringes a minus cosine response (the CDW is shown to have a pure minus cosine response in Ref. [25]). We then identify the "effective" start time which gives the waveforms this plus or minus cosine form, and record this as our measured delay time. We want to emphasize that this simple approach accurately captures the shift in delay times that is clearly visible in the raw data (longer delays at lower order fringes). The results are shown in Fig. 4(a). The higher-order fringes (4-10) need little adjustment to achieve the ideal phase relation, because they are generated instantaneously upon laser heating, while the first three fringes demonstrate a lag time that is close to the known hot-electron-phonon relaxation time in $\mathrm{Cr}(0.4 \mathrm{ps})$ extracted from transient IR reflection [28]. It should be noted that for the highest-order fringes, the periods and phase adjustments become smaller than the experimental timing uncertainty, so they are of limited significance, and our assumption that they are also created through the CDW mechanism does not affect the main findings here. The lag time decreases with frequency, so that the longest-wavelength phonons take the longest to populate. This is primarily due to the wave vector/frequency dependence of electron-phonon interactions, as discussed below. From a real-space view, establishing a longer-wavelength mode requires a collective rearrangement of atoms at longer distances, which will occur at the (finite) speed of sound. In principle there could be anharmonic phonon-phonon interactions driving the delay, but we show below that this effect is negligible.

In order to dissect the ultrafast population and depopulation of electronic and phononic states in $\mathrm{Cr}$, we perform first-principles simulations using the ABINIT software package [29], which implements density functional theory (a)

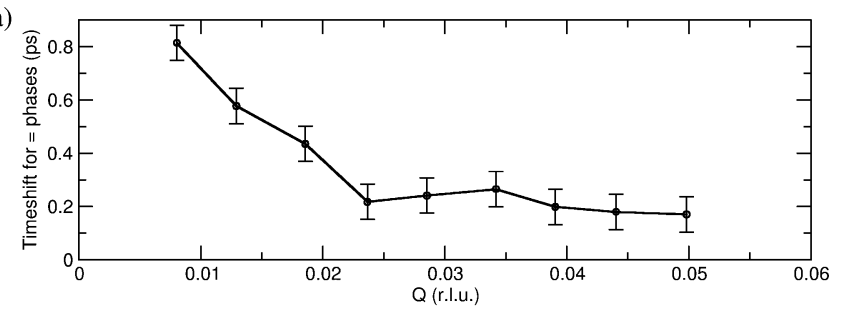

(b)

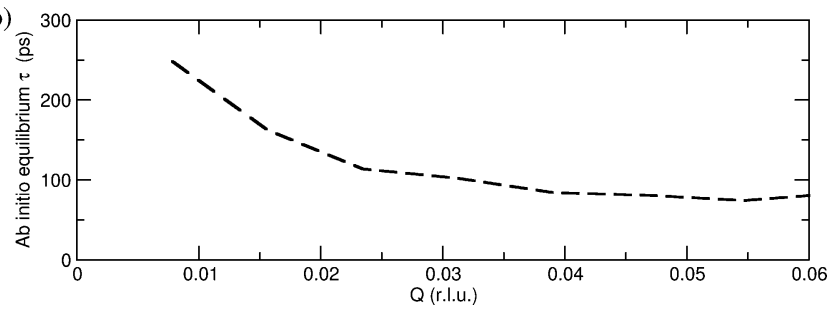

FIG. 4. (a) Experimental phonon generation lag times: The time by which $t=0$ must be shifted for each fringe to achieve the phase relation we expect. (b) Calculated equilibrium lifetimes for the longitudinal acoustic phonon mode in $\mathrm{Cr}$ (AFM GGA solution), for small $\vec{q}$ along the high-symmetry $\Gamma-H$ line.

(DFT). Phonons and the electron-phonon coupling (EPC) are calculated within the density functional perturbation theory [30,31]. See Supplemental Material (SM) for computational details [32].

The structural properties of $\mathrm{Cr}$ are already fairly described by spin-free DFT, but the antiferromagnetic (AFM) state is more stable $(40 \mathrm{meV})$. The phonon frequencies are well represented in all cases, with indistinguishable acoustic branches and slightly better zone-edge modes in the AFM generalized gradient approximation (GGA). The Fermi surface is, however, more delicate and critical for EPC (see SM [32] for details). We calculate the Eliashberg coupling constant $\lambda$, which integrates the coupling of all phonons with all electrons, and find $\lambda \simeq 0.056(0.313)$ and $\lambda\left\langle\omega^{2}\right\rangle \simeq 51$ (298) $\mathrm{meV}^{2}$ for the AFM (nonmagnetic) results. The EPC was experimentally found by Dresselhaus et al. [28] to be $\lambda$ of 0.13 and $\lambda\left\langle\omega^{2}\right\rangle \sim$ $128 \mathrm{meV}^{2}$. We note that these values are very sensitive to the exchange correlation, due to the complex spin/CDW ground state, and we confirm the comments in Ref. [28]: Neglecting spin overestimates the scattering. We have thus bracketed the global equilibrium EPC: The AFM model shows too weak EPC while the nonmagnetic one is too strong-the true magnetic structure and fermiology are in between.

The coupling of specific phonons to electrons at equilibrium is quantified by the inverse of the phonon linewidth [42]. These are plotted as a function of wave vector $\vec{q}$ in Fig. 4(b). There is a universal phase-space restriction near the zone center: For small $\vec{q}$, the intersection of the phonon sphere with the Fermi surface becomes a circle of decreasing diameter. This leads to the observed lifetime increase at low $\vec{q}$, both in calculations and experiment.

The equilibrium lifetimes are two to three orders of magnitude larger than the impulsive time delays measured in experiment. Strongly enhanced EPC in systems far from equilibrium has been reported many times, e.g., in bismuth [19,43], simple metals [44], and in electron diffraction experiments [45]. A 
central effect is the high local temperature occupying the electronic density of states (DOS) after a laser pulse. Following Lin $e t$ al. [44] we find a resulting increase of between 5 and 10 in the average EPC coupling in Cr. A related expression by Allen [see Ref. [46], Eq. (14)] yields temperature relaxation times of a few ps for electronic temperatures of few $1000 \mathrm{~K}$ (see SM [32]). The remaining difference with experiment is due to "true" nonequilibrium changes of the coupling matrix element itself. To recap, the response time order of magnitude is dictated by nonequilibrium effects, but its $\vec{q}$ dispersion is similar to equilibrium lifetimes, following a universal "phasespace" relation.

To quantify the importance of anharmonicity, phononphonon linewidths are calculated using the temperaturedependent effective potential technique [47]. Our calculations show that the these linewidths are at least one order of magnitude smaller than the EPC ones, and can be safely neglected.

In summary, we have demonstrated the generation of phonons in $\mathrm{Cr}$ via ultrafast optical excitation attributed to two distinct mechanisms - the releasing of a frozen CDW, and the generation of a coherent acoustic strain pulse. The CDW phonons have a response that is instantaneous upon heating of the electronic system, whereas those associated with the acoustic pulse demonstrate a generation lag which depends on the phonon frequency. Through phase-space considerations and calculations, we show that this frequency dependence is driven by the variable electron-phonon coupling strength, and has the expected $\vec{q}$ dependence, but is three orders of magnitude faster than predicted by equilibrium calculations. This points to a greatly enhanced scattering rate when the hot electrons are out of equilibrium with the lattice, which could be explored experimentally by varying the laser fluence and observing the effect on the phonon generation times.
A nonequilibrium description of the EPC should be able to quantify the observed electron-phonon coupling magnitudes in the future.

The work at UCSD was supported by the US Department of Energy, Office of Science, Office of Basic Energy Sciences, under Contracts No. DE-SC0001805 (ultrafast X-ray scattering, A.S., R.K., J.W., and O.G.S.) and No. DESC0003678 (thin film synthesis and characterization, S.K.K.P., R.K., V.U., and E.E.F.). O.M.M., B.M.M., and S.F. thank the Bundesministerium für Bildung und Forschung (BMBF) for financial support via Project No. 05K13FK2/05K16FK1. Use of the Linac Coherent Light Source (LCLS), SLAC National Accelerator Laboratory, is supported by the US Department of Energy, Office of Science, Office of Basic Energy Sciences under Contract No. DEAC02-76SF00515. This research used resources of the Advanced Photon Source (APS), a US Department of Energy (DOE) Office of Science User Facility operated for the DOE Office of Science by Argonne National Laboratory under Contract No. DE-AC02-06CH11357. We would like to acknowledge the support we received from Alec Sandy and Eric Dufresne of APS. M.J.V. acknowledges support from ARC grant AIMED (G.A. 15/19-09). Computational resources have been provided by the Consortium des Equipements de Calcul Intensif (CECI) (FNRS G.A. 2.5020.11); the Zenobe Tier-1 (Walloon Region G.A. 1117545); and by a PRACE-3IP DECI grants 2DSpin and PyLight on Beskow (G.A. 653838 of H2020). This publication is based upon work from COST Action TUMIEE (CA17126, European Cooperation in Science and Technology). A.H.R. acknowledges the support of NSF DMREF-1434897, OAC1740111 and US Department of Energy, Office of Basic Energy Sciences, Award DE-SC0019491.
[1] W. Shaw and L. Muhlestein, Phys. Rev. B 4, 969 (1971).

[2] M. F. DeCamp, D. A. Reis, D. M. Fritz, P. H. Bucksbaum, E. M. Dufresne, and R. Clarke, J. Synchrotron Radiat. 12, 177 (2005).

[3] B. M. Murphy, H. Requardt, J. Stettner, J. Serrano, M. Krisch, M. Müller, and W. Press, Phys. Rev. Lett. 95, 256104 (2005).

[4] M. J. Stern, L. P. René de Cotret, M. R. Otto, R. P. Chatelain, J.-P. Boisvert, M. Sutton, and B. J. Siwick, Phys. Rev. B 97, 165416 (2018).

[5] H. Park, X. Wang, S. Nie, R. Clinite, and J. Cao, Phys. Rev. B 72, 100301(R) (2005).

[6] S. Nie, X. Wang, H. Park, R. Clinite, and J. Cao, Phys. Rev. Lett. 96, 025901 (2006).

[7] T. Chase, M. Trigo, A. Reid, R. Li, T. Vecchione, X. Shen, S. Weathersby, R. Coffee, N. Hartmann, D. Reis et al., Appl. Phys. Lett. 108, 041909 (2016).

[8] D. A. Reis and A. M. Lindenberg, Light Scattering in Solids IX (Springer, Berlin, 2006), pp. 371-422.

[9] M. Trigo, J. Chen, V. H. Vishwanath, Y. M. Sheu, T. Graber, R. Henning, and D. A. Reis, Phys. Rev. B 82, 235205 (2010).

[10] D. Zhu, A. Robert, T. Henighan, H. T. Lemke, M. Chollet, J. M. Glownia, D. A. Reis, and M. Trigo, Phys. Rev. B 92, 054303 (2015).

[11] M. Chollet, R. Alonso-Mori, M. Cammarata, D. Damiani, J. Defever, J. T. Delor, Y. Feng, J. M. Glownia,
J. B. Langton, S. Nelson et al., J. Synchrotron Radiat. 22, 503 (2015).

[12] M. Trigo, M. Fuchs, J. Chen, M. Jiang, M. Cammarata, S. Fahy, D. Fritz, K. Gaffney, S. Ghimire, A. Higginbotham et al., Nat. Phys. 9, 790 (2013).

[13] A. Singer, S. K. K. Patel, R. Kukreja, V. Uhlîr, J. Wingert, S. Festersen, D. Zhu, J. M. Glownia, H. T. Lemke, S. Nelson, M. Kozina, K. Rossnagel, M. Bauer, B. M. Murphy, O. M. Magnussen, E. E. Fullerton, and O. G. Shpyrko, Phys. Rev. Lett. 117, 056401 (2016).

[14] K. Gaffney and H. Chapman, Science 316, 1444 (2007).

[15] T. Henighan, M. Trigo, S. Bonetti, P. Granitzka, D. Higley, Z. Chen, M. P. Jiang, R. Kukreja, A. Gray, A. H. Reid, E. Jal, M. C. Hoffmann, M. Kozina, S. Song, M. Chollet, D. Zhu, P. F. $\mathrm{Xu}$, J. Jeong, K. Carva, P. Maldonado, P. M. Oppeneer, M. G. Samant, S. S. P. Parkin, D. A. Reis, and H. A. Dür, Phys. Rev. B 93, 220301(R) (2016).

[16] D. M. Fritz, D. Reis, B. Adams, R. Akre, J. Arthur, C. Blome, P. Bucksbaum, A. L. Cavalieri, S. Engemann, S. Fahy et al., Science 315, 633 (2007).

[17] T. Saito, O. Matsuda, and O. B. Wright, Phys. Rev. B 67, 205421 (2003).

[18] C. Thomsen, H. T. Grahn, H. J. Maris, and J. Tauc, Phys. Rev. B 34, 4129 (1986). 
[19] Y. Giret, A. Gellé, and B. Arnaud, Phys. Rev. Lett. 106, 155503 (2011).

[20] D. Sangalli and A. Marini, Europhys. Lett. 110, 47004 (2015).

[21] H. J. Zeiger, J. Vidal, T. K. Cheng, E. P. Ippen, G. Dresselhaus, and M. S. Dresselhaus, Phys. Rev. B 45, 768 (1992).

[22] K. Ishioka and O. V. Misochko, Progress in Ultrafast Intense Laser Science (Springer, Berlin, 2010), pp. 23-46.

[23] P. Ruello and V. E. Gusev, Ultrasonics 56, 21 (2015).

[24] E. Fawcett, Rev. Mod. Phys. 60, 209 (1988).

[25] A. Singer, M. J. Marsh, S. H. Dietze, V. Uhlîr, Y. Li, D. A. Walko, E. M. Dufresne, G. Srajer, M. P. Cosgriff, P. G. Evans, E. E. Fullerton, and O. G. Shpyrko, Phys. Rev. B 91, 115134 (2015).

[26] A. Singer, S. K. K. Patel, V. Uhlîr, R. Kukreja, A. Ulvestad, E. M. Dufresne, A. R. Sandy, E. E. Fullerton, and O. G. Shpyrko, Phys. Rev. B 94, 174110 (2016).

[27] C. Kittel, Introduction to Solid State Physics (Wiley, Hoboken, NJ, 2005).

[28] S. D. Brorson, A. Kazeroonian, J. S. Moodera, D. W. Face, T. K. Cheng, E. P. Ippen, M. S. Dresselhaus, and G. Dresselhaus, Phys. Rev. Lett. 64, 2172 (1990).

[29] X. Gonze, F. Jollet, F. A. Araujo, D. Adams, B. Amadon, T. Applencourt, C. Audouze, J.-M. Beuken, J. Bieder, A. Bokhanchuk, E. Bousquet, F. Bruneval, D. Caliste, M. Côté, F. Dahm, F. Da Pieve, M. Delaveau, M. Di Gennaro, B. Dorado, C. Espejo et al., Comput. Phys. Commun. 205, 106 (2016).

[30] S. Y. Savrasov and D. Y. Savrasov, Phys. Rev. B 54, 16487 (1996).

[31] M. Verstraete and Z. Zanolli, in Computing Solids: Models, Ab-initio Methods and Supercomputing, edited by S. Blügel, N. Helbig, V. Meden, and D. Wortmann, Lecture Notes of the 45th Spring School 2014, Schlüsseltechnologien
Key Technologies Vol. 35, Forschungszentrum Jülich (Schriften des Forschungszentrums Jülich, Jülich, 2014), pp. C2.1-C2.29.

[32] See Supplemental Material at http://link.aps.org/supplemental/ 10.1103/PhysRevB.102.041101 for computational details, which includes Refs. [29,31,33-41].

[33] J. Trampenau, W. Petry, and C. Herzig, Phys. Rev. B 47, 3132 (1993).

[34] D. R. Hamann, Phys. Rev. B 88, 085117 (2013).

[35] M. van Setten, M. Giantomassi, E. Bousquet, M. Verstraete, D. Hamann, X. Gonze, and G.-M. Rignanese, Comput. Phys. Commun. 226, 39 (2018).

[36] J. P. Perdew, K. Burke, and M. Ernzerhof, Phys. Rev. Lett. 77, 3865 (1996).

[37] A. Eiguren and C. Ambrosch-Draxl, Phys. Rev. B 78, 045124 (2008).

[38] F. Giustino, Rev. Mod. Phys. 89, 015003 (2017).

[39] H. Møller and A. Makintosh, Inelastic Scattering of Neutrons (IAEA, Vienna, 1965).

[40] G. Kresse and J. Furthmüller, Phys. Rev. B 54, 11169 (1996).

[41] S. Cottenier, B. D. Vries, J. Meersschaut, and M. Rots, J. Phys.: Condens. Matter 14, 3275 (2002).

[42] G. Grimvall, Phys. Scr. 14, 63 (1976).

[43] J. Faure, J. Mauchain, E. Papalazarou, M. Marsi, D. Boschetto, I. Timrov, N. Vast, Y. Ohtsubo, B. Arnaud, and L. Perfetti, Phys. Rev. B 88, 075120 (2013).

[44] Z. Lin, L. V. Zhigilei, and V. Celli, Phys. Rev. B 77, 075133 (2008).

[45] L. Waldecker, R. Bertoni, R. Ernstorfer, and J. Vorberger, Phys. Rev. X 6, 021003 (2016).

[46] P. B. Allen, Phys. Rev. B 36, 2920 (1987).

[47] O. Hellman, P. Steneteg, I. A. Abrikosov, and S. I. Simak, Phys. Rev. B 87, 104111 (2013). 